\title{
Experiential Learning: Beyond the Classroom and Connecting with the Industry
}

\author{
Waqar Haque \\ University of Northern British Columbia, Canada \\ waqar.haque@unbc.ca
}

\begin{abstract}
To address dynamic needs of the industry, student learning must extend beyond the classroom. Considering the packed nature of traditional curriculum and logistics, incorporating new courses is challenging. A model is presented where the desired learning is acquired by engaging students in research partnerships, and without unduly extending the duration of their degree program. The success of this model has been demonstrated over many years by deployment of a variety of solutions for a diverse group of stakeholders, and an ever-growing demand for expansion. A few selected projects are presented in this paper to illustrate the skills acquired by students which include training in state-of-the-art technologies, creative thinking, research publications, and prospects for immediate hiring upon graduation. The benefits to faculty and industrial partners are also highlighted. Finally, the paper presents the challenges encountered which range from space, turnovers, transitioning, data access and perception of shadow IT. The ideas presented are applicable to all emerging areas.
\end{abstract}

Keywords. Experiential learning, emerging ICT areas, analytics, business intelligence.

\section{Introduction}

The rapidly changing needs of the industry and desire to recruit students trained in state-of-the-art technologies raises pedagogical challenges for the academia. At the same time, students face recruitment challenges upon graduation when they discover that the conventional training during their academic pursuits does not align with industry needs. It is difficult to incorporate the dynamic emerging areas of computer science (CS) in the curriculum. The reasons are twofold: first, the CS curriculum is packed with traditional courses leaving very little room for electives; second, the field is moving too fast and the demands change too rapidly to be fulfilled through coursework. The process of introducing new courses requires several layers of approval and is not conducive to the changing needs of the industry. Some examples of the desirable areas of training are Business Intelligence (BI), advanced analytics, cloud computing, Internet of Things (IoT) and Business Process Management (BPM). Both BI and BPM focus on increasing business value through analysis and optimization.

We have solved this problem by engaging community and industrial partners in collaborative projects which not only serve as proofs of concept, but have been 
successfully deployed in production mode. The projects provided pedagogical and financial benefits to the students, led to dissemination of results to research community, and opened avenues to increase business value within partner organizations. Some of the key challenges in undertaking these projects include availability of space, access to data, student turnover and resistance from the IT departments. In some healthcare related projects, privacy and confidentiality of data is also a key constraint and requires signing of non-disclosure agreements. In contrast, data for service-oriented projects is normally already in the public domain and does not require such clearances.

In this paper, we present some selected projects covering broad arenas including internal institutional tasks, conventional sectors such as healthcare, and nonconventional areas such as agriculture. The focus of these projects is on analytics, with or without the use of multi-dimensional Online Analytical Processing (OLAP) cubes. However, the ideas presented can be extended to other emerging areas as well.

\section{Related Work}

In rapidly evolving fields such as computer science, the academic institutions generally lag behind the industry needs. Many organizations therefore engage in inhouse R\&D and end up developing de facto standards in a race for the lead. A skill requirements review in the general field of information systems revealed that the "rapidly changing field requires the professional to continuously change as well" [1]. Analytics has become an increasingly strategic tool for companies to enhance their business value. A framework for integrated instruction of analytics, decision support and business intelligence using real cases with real data provides an opportunity for students to acquire the BI skills needed by companies [2]. The situation of ICT industry in EU and lack of ICT specialists trained in the required competences is presented in [3]. The authors clearly articulate a case for reengineering of the existing CS curriculum. A model curriculum has also been proposed to incorporate the emerging areas of business intelligence and business analytics [4]. This curriculum encompasses a hybrid academic domain of disciplines including statistics, management science, computer science and business information systems.

Academic institutions have tried to respond to industry needs in somewhat unstructured ways. Some have created new streams and programs whereas others have selectively integrated some areas into existing curriculum. These include BI, advanced analytics and automated business process management. Institutions have also resorted to offering certificates and diplomas through their Continuing Studies extensions. A comprehensive survey of Canadian and US schools is presented in [5].

\section{Opportunities}

The opportunities for students are broad, both within and outside of the academic institution. The limiting factor is generally space and time. The success of our approach has been demonstrated over a period spanning more than fifteen years. In 
this section, we present a few representative projects which have been successfully completed and deployed. The training provided through these projects span the general area of Business Intelligence (BI) including advanced analytics (Fig 3). The students not only learn how to design interfaces, but also engage in fairly sophisticated backend design which includes integration, analysis through multidimensional queries, and reporting on a variety of metrics. In addition, they have used extensions such as ASP.NET wrappers for rendering reports, and developing crossplatform capabilities. Finally, understanding the process of requirements analysis and benefits of agile development comes as a very desirable aspect of training.

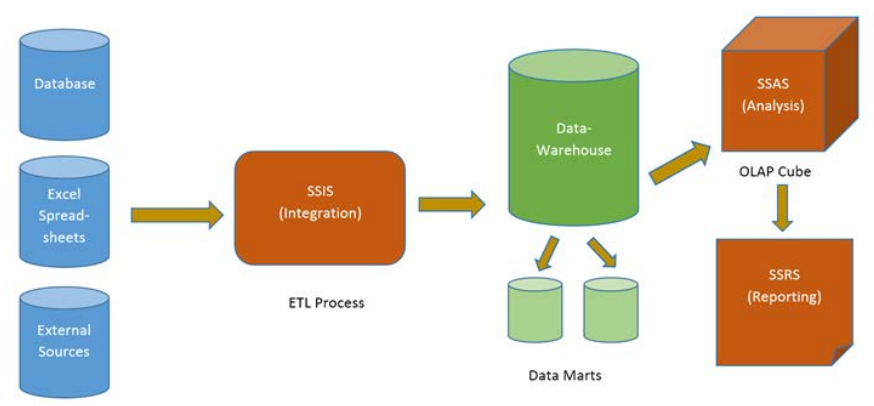

Fig. 1. BI Modeling Overview [6]

\subsection{Industrial Collaborative Research Projects}

A variety of industrial partners have been engaged ranging from silviculture and bioenergy to healthcare thus representing diverse requirements leading to evolution of creative techniques to deliver the unique objectives. The environment has also served as a platform for both students and potential employers to evaluate each other, resulting in recruitment prior to, or within days of, graduation.

A couple of representative projects completed using public domain data are related to health services delivery and availability of critical care services in geographically sparse areas. Healthcare organizations gather large volumes of data which is traditionally stored in legacy formats making it difficult to analyze or use effectively. The quality of this data is generally poor as it suffers from inconsistencies and lacks integrity. By applying BI techniques, we have been able to derive significant value from this data and made it available to broader audience through comprehensible and user-friendly interfaces (Fig 4). The process started with developing an integration process to cleanse and import data from disparate sources into a data warehouse. A 12-dimensional OLAP cube was then built to allow slicing along multiple dimensions determined by various key performance indicators (KPIs) representing population and patient profiles, case mix groups and healthy community indicators. In addition to an extensive suite of standardized reports, capabilities for ad hoc reporting on facility comparison and availability of various service levels was also provided. This data visualization and ability to slice-and-dice data to desired granularity provided the key benefits: the ability to make informed decisions for optimal resource allocation, and enhanced patient care. The use of mapping tools, customized shape files and 
embedded objects further augmented the navigation (Fig 5). Finally, web forms provide a mechanism for remote uploading of data and transparent processing of the cube in real-time. Access controls were implemented where necessary. The modular design left hooks for expansion without the need to rebuild the entire system.

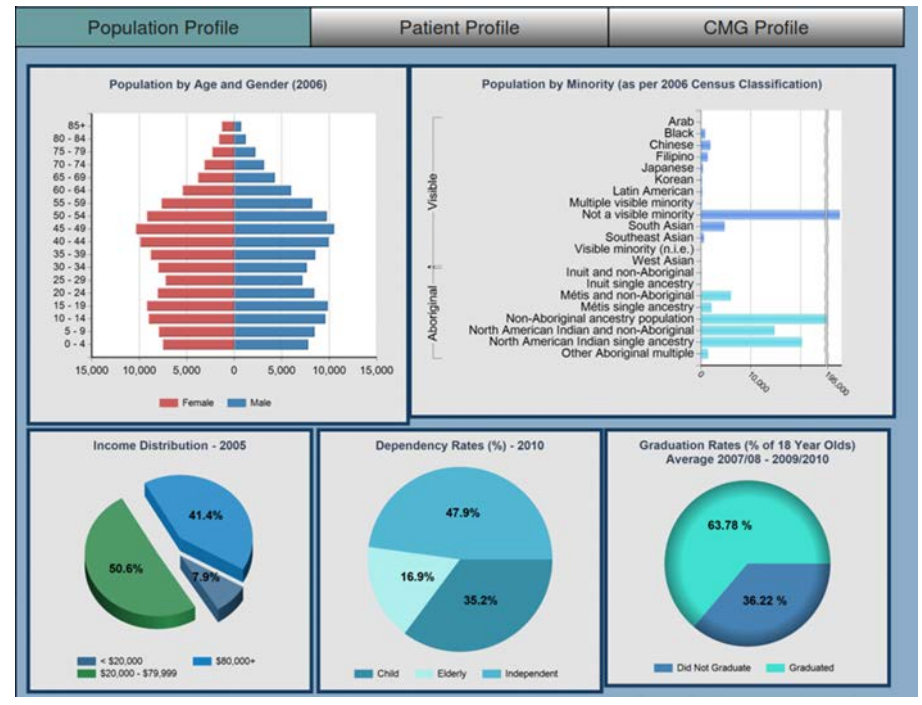

Fig. 2. Services Main Dashboard: Population Profile [10]

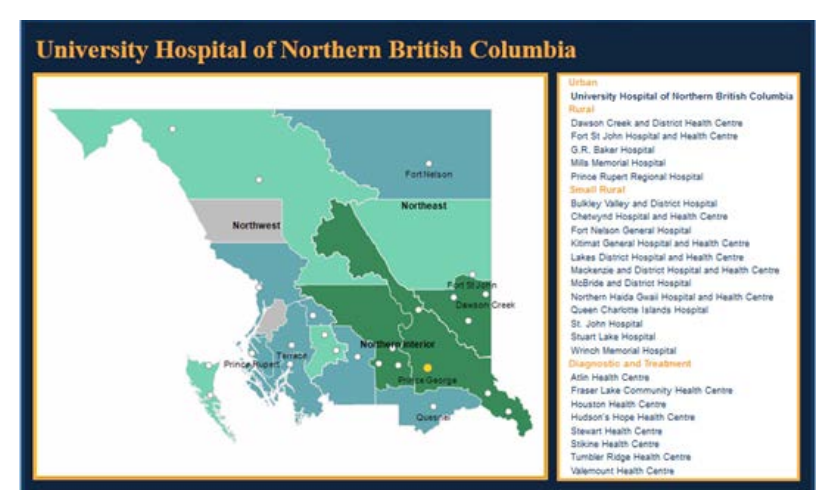

Fig. 3. Navigation Map Interface [7]

In many cases, patients suffer from medical conditions that can often be treated outside of a hospital setting. These conditions are commonly known as Ambulatory Care Sensitive Conditions (ACSC). The preventable hospitalizations can result in reduced healthcare costs and improved patient care. A BI model was developed to provide analytics based on highly customizable scenarios of population and medical records. The parameterized reports allowed selection of multiple dimensions simultaneously and were rendered in a matter of seconds.Fig. Fig 6 shows the main 
page from which intuitive navigation leads to other reports. This work was later extended to involve advanced analytics where predictive models were built to forecast 3-5 years in the future. Such information is very useful for reallocation of resources, if deemed necessary.

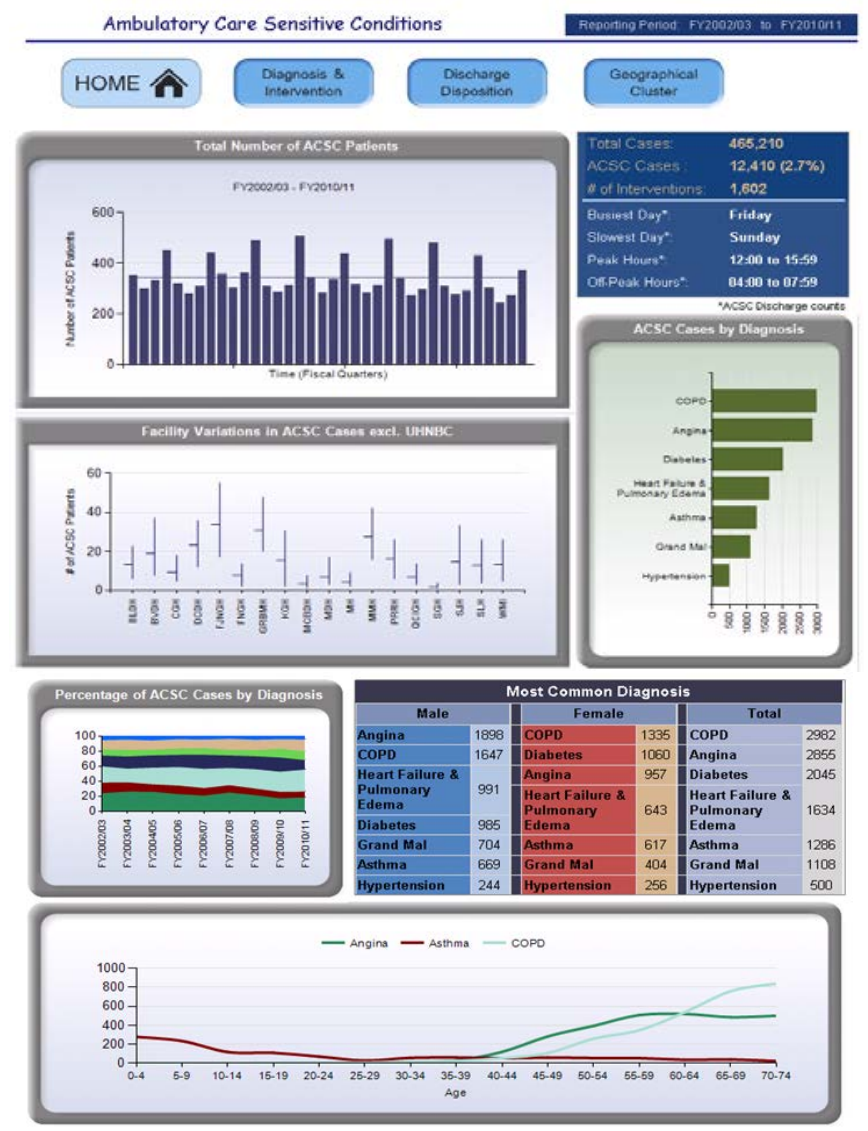

Fig. 4. ACSC - Diagnosis Grouping [8]

Often patients are transferred within facilities for several reasons. In such cases, the transfer operator must be able to objectively identify a destination which meets the needs of a patient, within the constraints of facility's limitations. In order to facilitate an optimized and efficient patient transfer, a visual interface accessing a single data warehouse and using BI techniques was designed. The interface included map based navigation of the health authority as well as an interactive filtering mechanism to determine facilities meeting the selected criteria. These facilities are then presented to the user sorted by distance, time and available transportation modes (ground, air, water). The data visualization is backed by an intuitive data entry web form which safely constrains the data while ensuring consistency. The overall time required to identify the optimal destination for inter-facility transfers is reduced from hours to a 
few minutes with this interactive solution [7]. The solution is currently being expanded to include all health authorities in the province.

To demonstrate the use of business intelligence and analytics in non-conventional areas, we developed a model using data that was collected over a number of years for tracking plantation and monitoring stand development. The data existed as legacy reports in the form of simple tables, line charts and scatter graphs which did not provide any strategic or intuitive insights. Since the data was never collected for the purpose of analytics, it presented the typical challenges including inconsistencies, missing data, and incompatible formats. OLAP techniques were once again used to transform this data into an interactive dashboard for querying, reporting and visualization (Fig 7) [9]. The proposed model has been used to link regenerated stands under various operational conditions with realistic long-term growth and yield predictions.

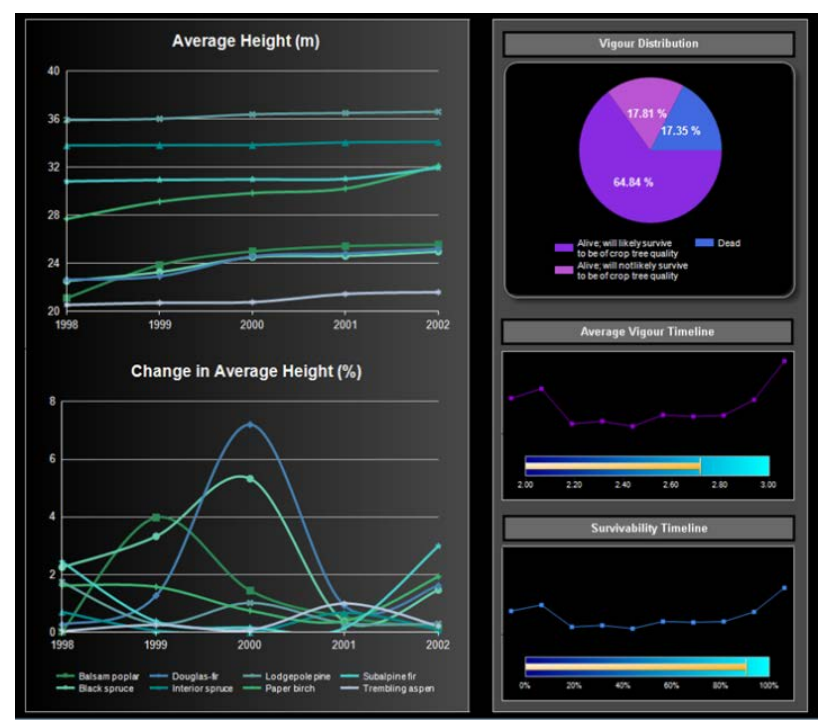

Fig. 5. Sample dashboard for vegetation management [9]

\subsection{Internal Projects}

The IT departments within academic institutions are overwhelmed with help desk and support activities. They rarely engage in state-of-the-art initiatives though the need for such projects is widespread. There are separate teams formed for institutional reporting using primitive methods such as spreadsheets and basic charts. This does not provide analytics for strategic objectives. This void can be covered by taking on proof of concept projects under the supervision of research faculty. Several projects have been completed over the last few years which resulted in direct training of a large number of undergraduate and graduate students. These projects have been in areas including enrolment management, workload assignments, tracking student progress, asset management, and automating complex tasks together with reporting. 


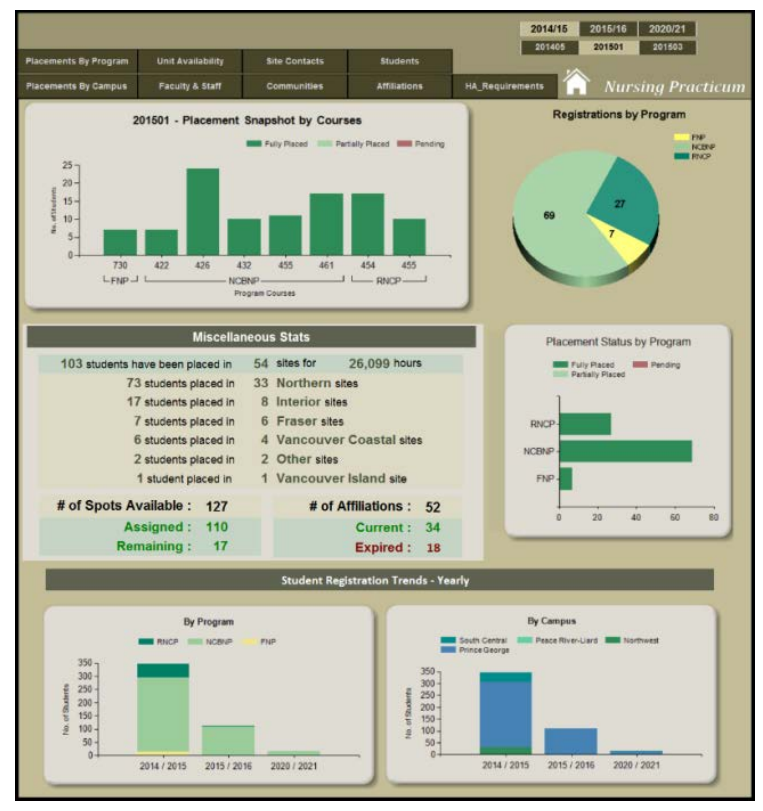

Fig. 6. Nursing Placement - Main Dashboard [10]

Placements for nursing practicums has been a challenging task because of the frequency, volume and number of facilities spanning vast geographical areas. The existing practice involved extensive use of spreadsheets, whiteboards and phone calls. BI techniques were used to design a sophisticated dashboard (Fig 8) which not only solved the placement problem, but also provided extensive reporting capabilities. In addition, a number of embedded controls were included to reduce errors such as oversubscribing and duplications. The system also auto-generated notifications for both students and coordinators. A suite of interactive web forms allows data to be entered by students (preferences), coordinators and managers. Historical data is also accessible for comparison purposes.

While OLAP cubes are commonly used to deliver business intelligence, this may be an overkill in situations which do not require multi-layered drill downs, but still need multi-dimensional analysis and reporting. This was demonstrated through several projects where a combination of web interfaces and business intelligence tools were used while bypassing OLAP cubes. One of these applications was an interface to track progress of graduate students through their programs. The key components of such a system are shown in Fig 9 whereas a snapshot of the top-level dashboard is shown in Fig 10. This interface allows efficient rendering of reports from a business intelligence tool stack, in combination with web forms for data integration. The elimination of the middle layer resulted in significant performance enhancement, rapid application development, and created stable, user-friendly, low-maintenance platform which requires minimal operational support from the IT department. 


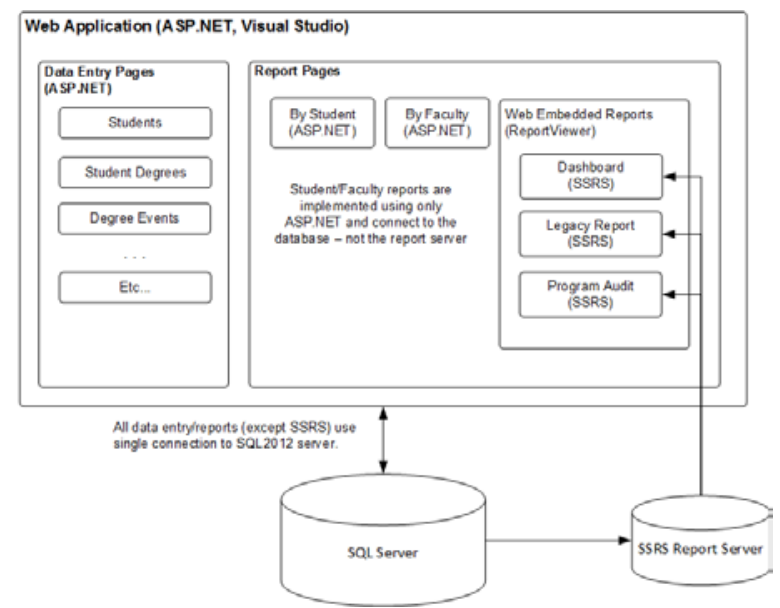

Fig. 7. Graduate Program Tracking: Components of the Framework [11]

\section{Challenges}

The success of the proposed model has been proven over a period spanning more than fifteen years. However, this did not come without challenges, both external and internal. When projects involve access to private and confidential data, arrangements should be made with the partners to anonymize the data and, in some cases, provide access to remote servers so that no data transfer is involved. These processes can be long and may need a champion in the partner organization to facilitate the process. Further, though the students are compensated when they work on these projects, there is no way to compete with the commercial rates. Thus, it is important to have informal anti-poaching arrangements with industrial partners as this is of mutual benefit for all involved. Internally, space and resources are an issue and it is important for the institution to recognize that this is a research initiative. Hosting of applications in the research lab should be avoided due to data confidentiality, licensing and support reasons. Selection of students is also important and a mix of both undergraduate and graduate students is essential. Overlapping teams should be structured keeping in mind that there will be continuous transition due to graduation. Students with business majors have also proven to be very effective in creating mocks and for frontend design. A technical review by partners must be conducted at an early stage to avoid any migration issues. 


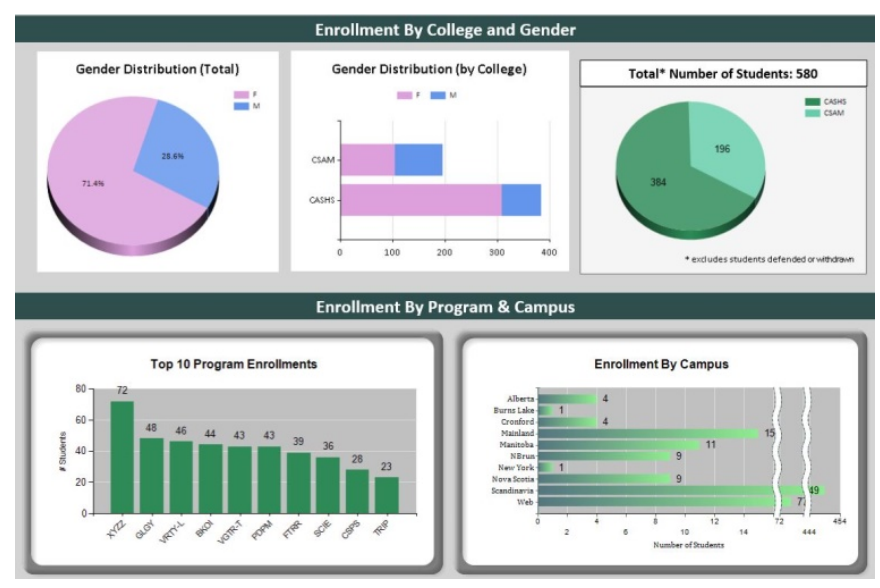

Fig. 8. Graduate Enrolment Snapshot [11]

\section{Conclusions}

Despite a surge in unfilled positions, students graduating with a conventional degree in computer science face challenges in the labour market. Unlike Coop programs, experiential learning during the course of academic program prepares students for the real-world job market without extending the duration of their studies. The model is equally successful for both graduate and undergraduate students. The benefits are multi-faceted: for supervising faculty, new tools and techniques are developed for dissemination to research community, and research grants become relatively easy to acquire; for students, they are able to co-author papers, get trained in state-of-the-art technologies and are quickly recruited; for partners, they get projects completed cost effectively and in a timely manner - some of these projects would not even have gotten off the ground due to organizational priorities. At the same time, they are able to scout and hire students who are already trained on their platforms. It should be emphasized that training under this model does not come without challenges which include space, data access, continuity and occasional loss of talent.

\section{References}

[1] D. Simon and K. Jackson, "A Closer Look at Information Systems Graduate Preparation and Job Needs: Implications for Higher Education Curriculum Enhancements," World Journal of Education, vol. 3, no. 3, pp. 52-62, 2013.

[2] B. Gupta and U. Raja, "Teaching Analytics, Decision Support, and Business Intelligence: Challenges and Trends," in Reshaping Society through Analytics, Collaboration, and Decision Support, Springer, 2015, pp. 205-209.

[3] M. Milosz and E. Lukasik, "Reengineering of computer science curriculum according to technology changes and market needs," in IEEE Global Engineering Education Conference (EDUCON), Tallinn, 2015. 
[4] M. Mitri and S. Palocsay, "Toward a Model Undergraduate Curriculum for the Emerging Business Intelligence and Analytics Discipline," Communications of the Association for Information Systems, vol. 37(31), 2015.

[5] W. Haque, "Incorporating Multidisciplinary Emerging Areas in CS Curriculum: the Pedagogical Challenges," in 21st Western Canadian Conference on Computing Education, Kamloops, 2016.

[6] W. Haque, B. Urquhart, E. Berg and R. Dhanoa, "Using Business Intelligence to Analyze and Share Health System Infrastructure Data in a Rural Health Authority," Journal of Medical Internet Research, vol. 2, no. 2, 2014.

[7] W. Haque, B. A. Derksen, D. Calado and L. Foster, "Using Business Intelligence for Efficient Inter-Facility Patient Transfer," in Information Technology and Communication in Health (ITCH), Victoria, 2015.

[8] W. Haque and J. A. Edwards, "Ambulatory Care Sensitive Conditions: A Business Intelligence Perspective," in Advances in Health Informatics Conference, York, 2012.

[9] W. Haque, J. Edwards and E. Belsham, "A Decision Analysis Model for Vegetation Management," in Int'l Conference on Information and Communication Systems, Amman, 2011.

[10] W. Haque, D. W. Ahmad, D. Calado, R. Dhanoa and M. E. Samani, "Business Intelligence Enabled Nursing Practicum Placements and Reporting," Journal of Advances in Information Technology, vol. 6, no. 3, pp. 118-123, 2015.

[11] W. Haque and R. Dhanoa, "A Framework for Reporting and Analytics without OLAP Cubes," in Multidisciplinary Academic Conference on Education, Teaching and Learning (MAC-ETL), Prague, 2015. 